\title{
THE INFLUENCE OF HIGH LATITUDE OFF-GREAT CIRCLE PROPAGATION EFFECTS ON HF COMMUNICATION SYSTEMS AND RADIOLOCATION
}

\author{
E.M. Warrington, A.J. Stocker and N.Y. Zaalov \\ Department of Engineering, University of Leicester, U.K.
}

\section{INTRODUCTION}

Owing to the presence of tilts and gradients in the high latitude electron density distribution, HF radio signals often arrive at the receiver over paths well displaced from the great circle direction. Deviations of a few degrees are associated with tilts due, for example, to the solar terminator and to travelling ionospheric disturbances (TIDs) [1]. Very large deviations are particularly prevalent in the high latitude regions where signals often arrive at the receiver with bearings displaced from the great circle direction by up to $\pm 100^{\circ}$ or more. These large deviations from the great circle path (GCP) are due to electron density depletion and the associated ionospheric tilts within the mid-latitude trough at sub-auroral latitudes $[2,3,4]$, whereas in the polar cap they are attributed to the presence of convecting patches and arcs of enhanced electron density [5].

Such large deviations of the direction of arrival of the signals from the GCP have serious implications for the operation of communication and radiolocation systems operating within the HF band. For communication systems, directional antennas are often employed and there is a significant possibility that performance will be degraded at times when the supported propagation path is at directions well displaced from the main lobe of one or both of the transmitting or receiving antennas. Radiolocation systems usually operate by measuring the direction of arrival at several receiving sites. The location of the transmitter is then estimated from the intersection of the individual lines of bearing from each receiving site. Deviations from the GCP will therefore adversely affect the estimate of the transmitter location. Although it is not thought possible to correct for these types of bearing error, it is possible to predict the periods during which the large deviations occur. These predictions could be used to assign ionospherically based weighting factors to the individual line of bearing measurements from a network of DF receiving stations in order to improve the accuracy of the position estimate.

In addition to the large scale tilts which cause gross deviations of the signal from the great circle direction, irregularities in the electron density distribution may be considered as providing a rough reflecting surface for HF radio waves. As a result of this roughness, signals associated with each propagation mode arrive at the receiver over a range of angles in both azimuth and elevation. Accordingly, a single ionospheric mode is often modelled as a single ray specularly reflected from a smooth ionosphere surrounded by a cone of rays produced by the roughness of the ionosphere. This model is an over-simplification in practice, particularly within the disturbed high latitude ionosphere, since it assumes a smooth ionosphere of infinite extent upon which are superimposed localised irregularities leading to the scattered energy, and no account is taken of larger scale, possibly time varying, effects.

The directional spread of the received signal energy is an important parameter to be considered in the design of multi-element receiving arrays and the associated signal processing methods used, for example, in radiolocation or adaptive reception systems. It is often assumed in such systems that the signal environment comprises a limited number of specularly reflected signals arriving at the antenna array from well defined directions. However, for high latitude paths, this is often not the case. On a polar cap path from Isfjord to Alert, for example, azimuthal standard deviations of up to $35^{\circ}$ were measured [6]. Furthermore, the effects tend to be frequency dependent with large differences in the direction of arrival and directional spread evident [7].

A common feature of high latitude HF propagation is the large Doppler and delay spreads imposed on the signal. The magnitude of these effects is such as to severely limit the data throughput achievable due to current technological limitations in modem design. The large Doppler spreads are usually associated with large directional spreading [6] and recent work [8] has indicated that adaptive beam / null steering from an array of antennas can be employed to exploit the directional spreading effects to reduce the apparent Doppler spread at the modem input. 
Various measurements of off great-circle propagation effects over a range of high latitude paths and their interpretation have been undertaken over a number of years by researchers at the University of Leicester. Reports of this work in the literature have been referred to above. The aim of this paper is to outline some of the important results of this research and to discuss work in progress aimed at further improving our understanding of the high latitude propagation mechanisms and at methods for taking the propagation effects into account when designing and operating HF radio systems. The descriptions of past work presented here are necessarily brief and reference should be made to the papers cited for more details.

\section{GROSS DEVIATIONS FROM THE GREAT CIRCLE DIRECTION}

\section{Paths along the mid-latitude trough}

The depleted electron densities and the electron density gradients associated with the mid-latitude trough region of the ionosphere (a sub-auroral feature) are responsible for large and prolonged deviations of the direction of arrival from the GCP. Example measurements of this type of propagation made over a one month period on a trans-Atlantic path from Canada to the UK are presented in Figure 1. These measurements clearly illustrate the magnitude of the effects (typically up to around $\pm 50^{\circ}$ ), the prolonged duration of the deviations (typically around 10 hours for this path) and also the relationship with the level of geomagnetic activity as given by the $A_{p}$ index.

A more detailed discussion of trough effects on this and other paths is given by Rogers et al [2]. Current research being undertaken by the University of Leicester is contained in elsewhere in these proceedings [4].
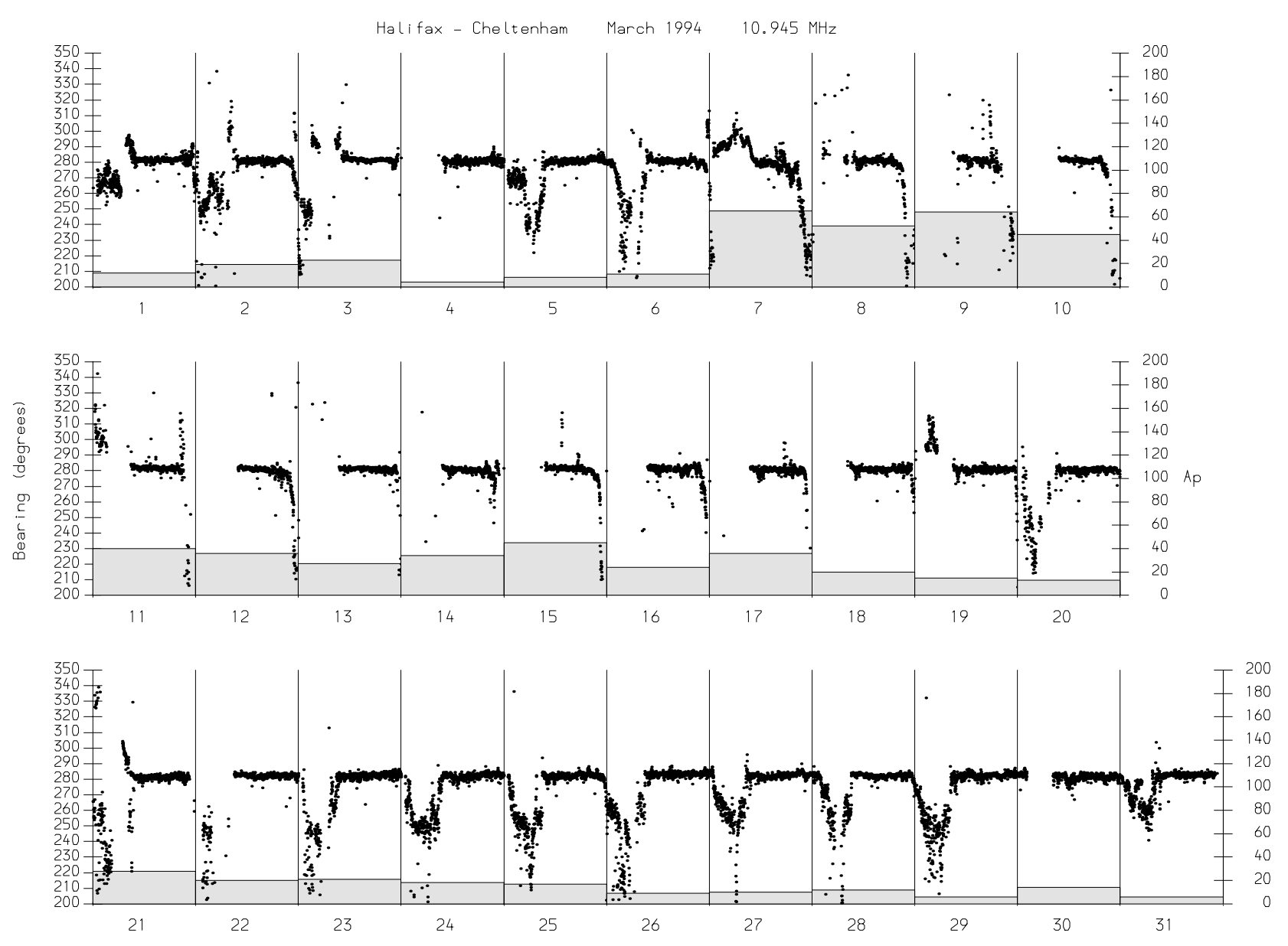

Figure 1. Bearings measured over the Halifax to Cheltenham path at $10.945 \mathrm{MHz}$ during March 1994 (the dates on these plots are for the UT day). Daily Ap values are also indicated. 


\section{Polar cap paths}

Observations over recent years have established that large scale electron density structures are a common feature of the polar cap F-region ionosphere. During periods of southward directed Interplanetary Magnetic Field (IMF) $\left(\mathrm{B}_{\mathrm{z}}<0\right)$ and the associated high levels of geomagnetic activity, patches of plasma 100-1000 km across with electron density enhancements of up to a factor of 10 above the background densities have been observed in the high latitude F-region ionosphere. These drift antisunwards across the central polar cap at velocities of a few kilometres per second in the high latitude convection current flows $[9,10]$. When geomagnetic activity is low and the IMF is directed northward (approximately $50 \%$ of the time), Sun-Earth aligned arcs of plasma with electron density enhancements of a factor of 2-3 above the background can occur. These plasma striations are elongated for thousands of kilometres in the trans-polar noon-midnight direction but are much narrower (around $100 \mathrm{~km}$ ) in the dawn-dusk direction. These features can persist for periods often in excess of one hour in the background F-region ionosphere [11] and have been found to be approximately twice as prevalent in the morning sector than in the evening sector [12]. They drift across the polar cap at velocities of a few hundred metres per second, generally in a duskward direction [10].

The electron density gradients associated with these large scale electron density structures form tilted reflection surfaces for HF radio waves which allow off great circle propagation paths to be established between the transmitter and the receiver. In order to investigate this type of propagation, a series of experiments [5] have been undertaken in which the bearings and signal characteristics of a number of HF transmissions were measured by means of a wide aperture goniometric DF system located at Alert in northern Canada (see Figure 2). Large quasi-periodic bearing variations of up to $\pm 100^{\circ}$ from the great circle direction were observed. Measurements over one of these paths will now be briefly considered.

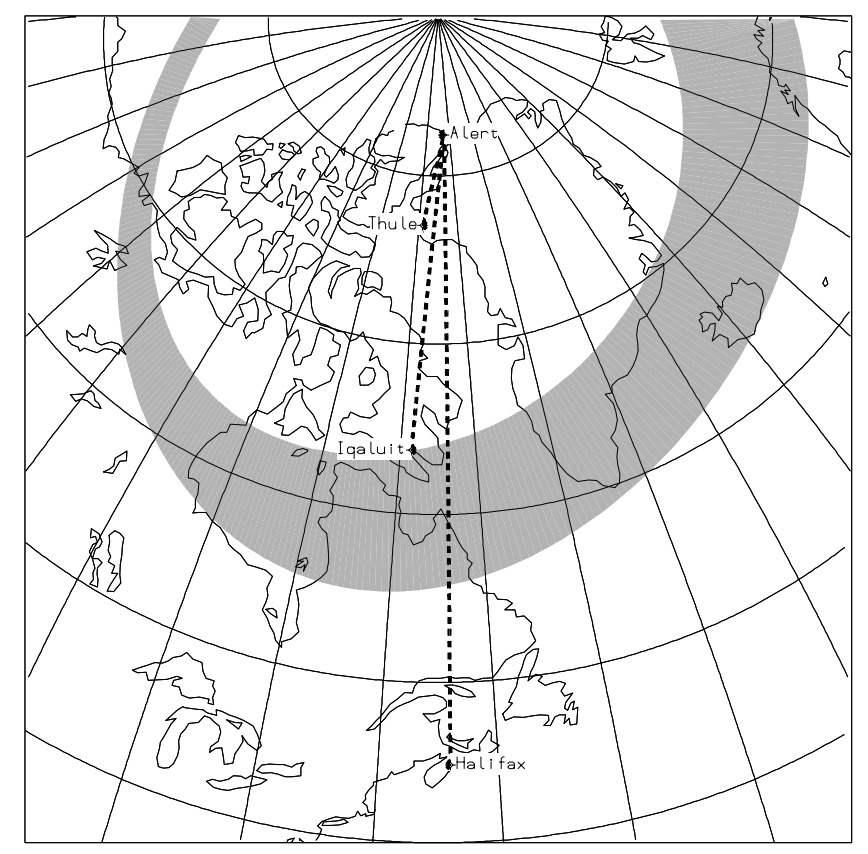

Figure 2. Location of the transmitting and receiver sites. A modelled position of the auroral zone at 0000 UT and $A_{p}=15$ is also shown [13].

\section{Iqaluit to Alert path}

The $2100 \mathrm{~km}$ path from Iqaluit to Alert is always contained within the polar cap ionosphere. In winter, the path remains in darkness for long periods and the reverse is true in summer. There is, consequently, a marked seasonal dependence of the signal behaviour with large $\left(\sim 100^{\circ}\right)$ bearing deviations observed during winter and equinoctial months and only small $\left(<10^{\circ}\right)$ fluctuations for most of the time during the summer.

There is an underlying diurnal trend for propagation to deviate to the west of the GCP (high bearing angles) in the evening sector (local midnight at the GCP mid-point is 0430 UT) with propagation returning from the 
east of the GCP (low bearing angles) in the morning. This may arise from very large scale ionospheric gradients in the polar cap associated with the solar terminator. More rapid bearing swings with periods of about 30 minutes are often superimposed upon these trends which are attributed to the presence of convecting patches or arcs of enhanced ionisation.

An example period illustrating the rapid bearing swings observed at $9.292 \mathrm{MHz}$ for the period 21-24 February 1994 is presented in Figure 3 together with values of the 3-hourly ap index and the $\mathrm{B}_{\mathrm{y}}$ and $\mathrm{B}_{\mathrm{z}}$ IMF parameters. A geomagnetic storm is evident on 21-22 February. The principal bearing swings on the night of the 21-22 - a period of southward IMF and high ap values - have a decreasing bearing angle and occur in the six hour period before local midnight (0430 UT) whereas the principal bearing swings on the following night - a period of northward IMF and low ap - have an increasing bearing and occur principally in the hours following local midnight.

When the IMF is directed southward $\left(\mathrm{B}_{z}<0\right)$, patches of ionisation drifting anti-sunwards would lead to a preponderance of decreasing bearing angle swings in the pre-midnight hours and increasing bearing angle swings in the hours after midnight. Figure 3 shows this to be the case where $\mathrm{B}_{\mathrm{y}}<0$, although where $\mathrm{B}_{\mathrm{y}}>0$ there is a distinct lack of decreasing swings in the pre-midnight sector. Reference to the expected convection flow [14] indicates that for these conditions the flow direction is skewed towards the evening sector and patches of solar produced ionisation will drift across the dawn side of the polar cap, thus producing the likelihood of a decreasing bearing angle swing. When the IMF is directed northwards $\left(B_{z}>0\right)$, the principal large scale electron density structures within the polar cap ionosphere are sun-aligned arcs [10]. A series of arcs drifting steadily across the polar cap from dawn to dusk would lead to the expectation that increasing bearing swings would be observed during the time sector 1800 to $0600 \mathrm{LT}$, with the largest swings expected in the midnight sector. Decreasing bearing swings would be observed in the local time sector 0600 to 1800 with the largest swings in the noon sector.

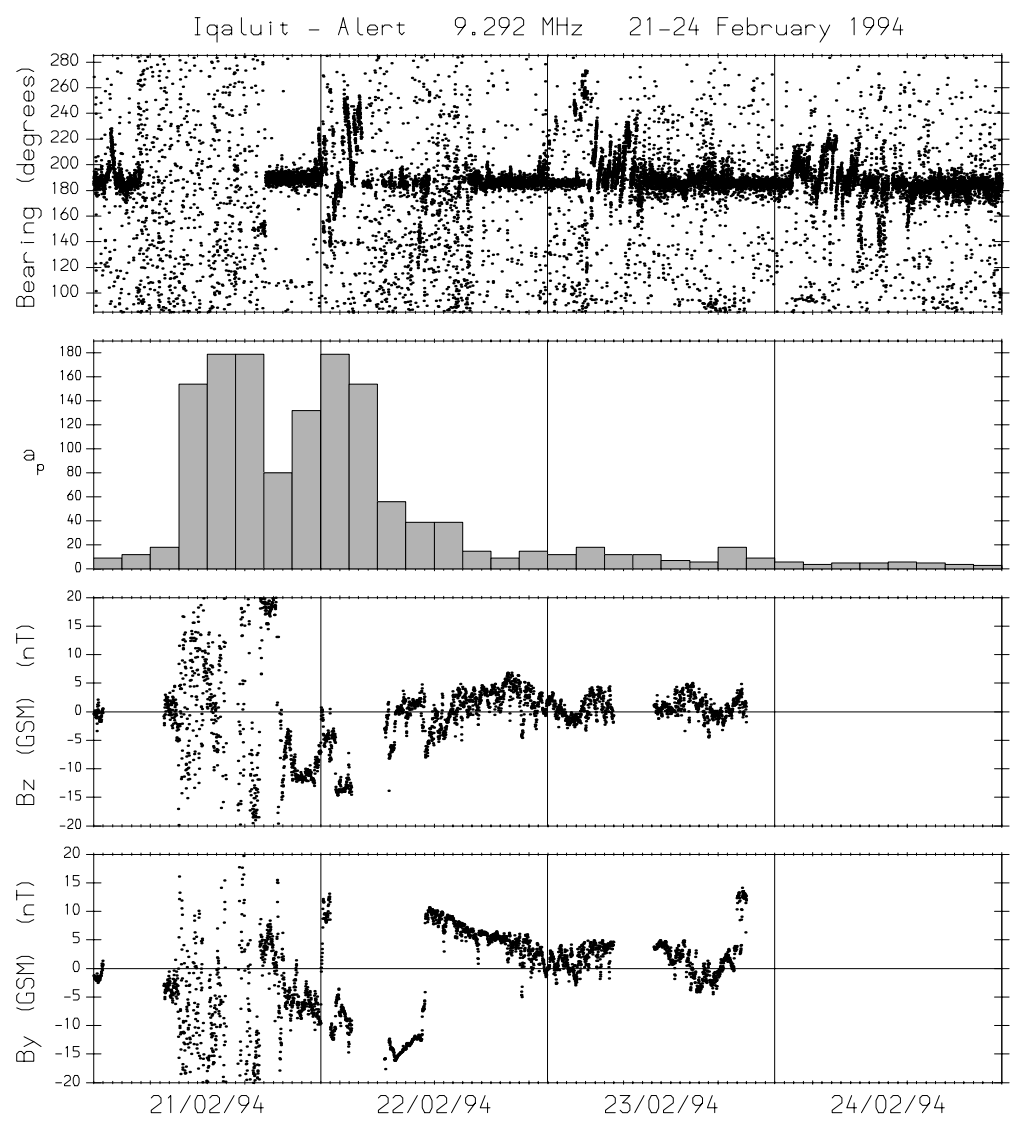

Figure 3. Bearings measurements for the $9.292 \mathrm{MHz}$ transmission from Iqaluit received at Alert for the period 21-24 February 1994. Three-hourly $a_{p}$ values and the IMF By and Bz values are also shown (middle and bottom panels). 


\section{Simulations}

Simulation studies are currently being undertaken in order (a) to provide confirmation that the presence of blobs and arcs in the polar cap ionosphere would produce the observed directional effects, and (b) importantly, the simulations will allow for the influence of the ionospheric effects on paths and frequencies other than those for which observations were made to be estimated.

A numerical code ray tracing code [15] is employed in the simulations in which the main parameters of the electron density model (critical frequency, critical height, and scale height of each layer) are based on values obtained from IRI model [16]. Localised, time varying, enhancements in the electron density profile were then applied to the model to represent the convecting patches and arcs.

An example simulation for a period dominated by the presence of arcs is presented in Figures 4 and 5. The first of these figures shows a simulated ionogram (note that the simulation was undertaken between 8 and $16 \mathrm{MHz}$, hence traces are not shown outside this frequency range) which is very reminiscent of those observed in practice (see [17]). The direction of arrival as a function of time for a simulated $10.45 \mathrm{MHz}$ signal propagating through this time-varying modelled ionosphere is presented in Figure 5 and is, again, very reminiscent of the observations.

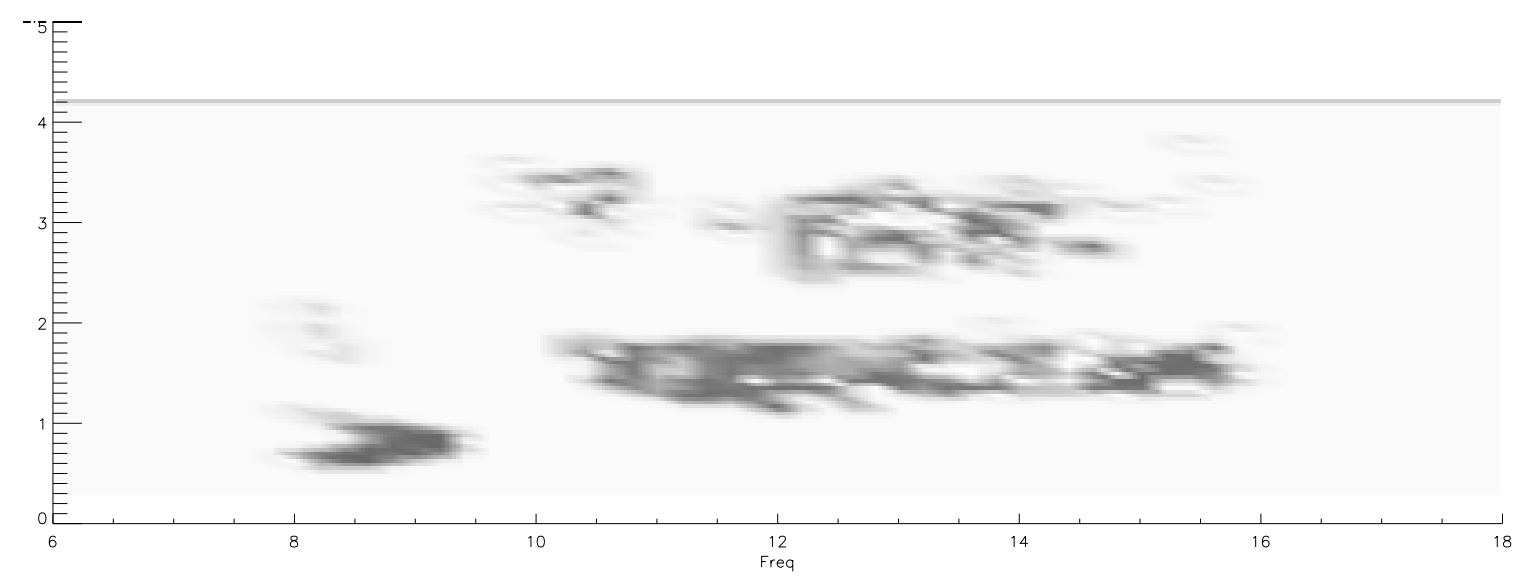

Figure 4. Ionogram produced by ray tracing simulations through an ionosphere containing arcs of enhanced electron density.

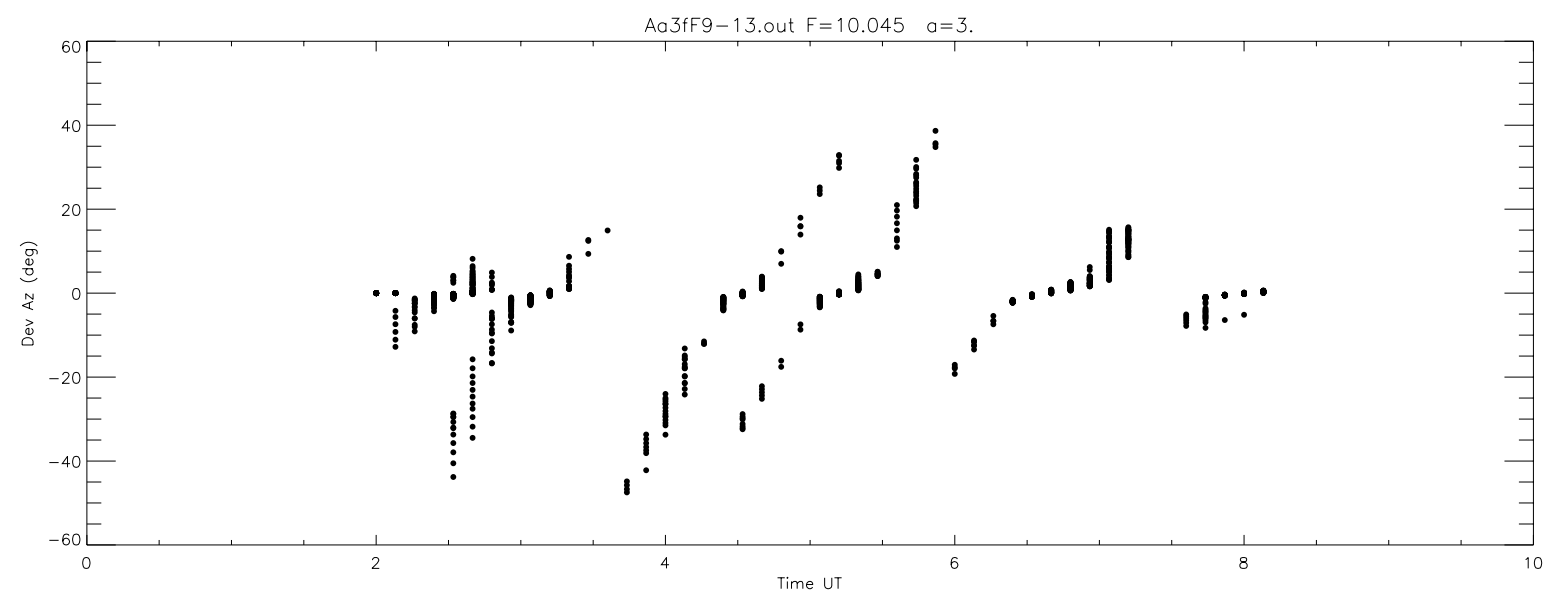

Figure 5. Time history of the direction of arrival (azimuth only) of a $10.45 \mathrm{MHz}$ signal propagating through a model ionosphere containing arcs of enhanced electron density. 


\section{SPATIALLY DIFFUSE REFLECTIONS}

The directional spread in the received signal energy is an important parameter to be considered in the design of multi-element receiving arrays and the associated signal processing methods used, for example, in direction finding or adaptive reception systems.

\section{Effect on conventional superresolution direction finding}

Measurements have been made with a superresolution direction finding (DF) system over the Iqaluit to Alert path [18] (this path has been mentioned previously in this paper in connection with measurements made with a goniometric DF system). A common feature of the measurements reported in [18] is the presence of multiple traces in the time history of the estimated bearings (see, for example, Figure 6) which are often not evident in the goniometric measurements made at the same time. When well separated, the multiple traces probably arise due to the presence of multiple reflectors. Closely separated multiple traces are also evident and whilst these may also arise from the presence of multiple reflectors (i.e. patches or arcs of enhanced ionisation) closely tracking one another (see Dumas [19]), it was found that the precise nature of the multiple traces is dependent both upon the array geometry and algorithm employed.
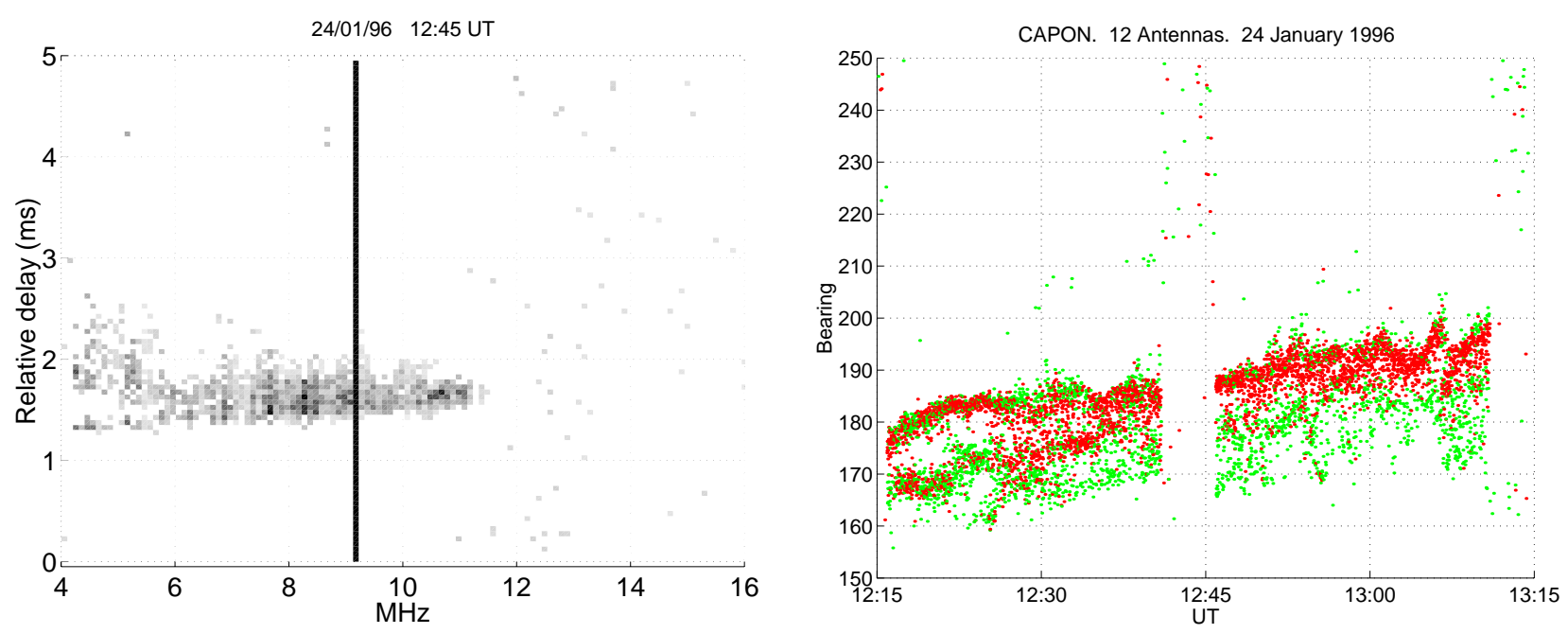

Figure 6. The left hand panel shows the oblique ionogram recorded over the Iqaluit - Alert path 12:45 UT on 24 January 1996. Bearing estimates obtained using a loaded CAPON estimator [20] for the period either side of the time of the ionogram are given in the right hand panel.

The often diffuse nature of the ionogram traces recorded over the same propagation path is indicative of a 'rough' ionospheric reflector containing irregularities which result in multiple intra-mode reflections causing the signal to arrive at the receiver over a range of directions in both azimuth and elevation. A modelling study has been undertaken (reported in [18]) in which simple diffuse reflection model was employed. Many of the features observed in the estimated bearings could be reproduced by means of this model (i.e. the presence of multiple, closely separated bearing traces tracking one another, and the algorithm and array geometry dependence). Differences in detail between the model results and the measurements occurred and were to be expected. These can readily be explained by the differences between the precise energy distributions included in the model and those which occurred in practice.

Many superresolution algorithms currently employed are based around the assumption that the signal environment comprises a small number of signals with planar wavefronts. It is evident that this is not well representative of the HF signal environment encountered in the high latitude regions and there is a clear need for new algorithm developments specifically tailored for these complex propagation conditions. In particular, it is essential that over-simplistic assumptions about the signal environment are not made. Read [21] describes two such algorithms currently under development: SML (Spread Maximum Likelihood) which models the signal environment as a number of raised cosine power distributions, and SPIRE (Spatial Incoherent Region Estimator) which attempts to estimate the energy distribution without making any prior 
assumptions about the distribution. With regard to these developments, it is noteworthy that the types of DF system employed in this study with appropriate processing algorithms are well suited to radio based investigations of the ionosphere and to investigations of HF radio propagation mechanisms for which estimates of the energy distribution are invaluable. This latter requirement differs markedly from radiolocation applications where a simple (but accurate) estimate of the great circle direction to the transmitter is the major requirement.

\section{Directional measurements of signals radiated by a channel sounding system}

A series of measurements [22, 23] have been made of the signals radiated by two of the DAMSON (Doppler And Multipath SOunding Network) transmitters [24]. This channel sounding system allows signal strength, time-of-flight, time dispersion due to multipath propagation and frequency dispersion (Doppler spread and Doppler shift) to be measured over point-to-point communications paths. All of these parameters are of great importance to the design and operation of HF communications equipment in the high latitude regions. In this regard, it should particularly be noted that, in general, data modems operate well under conditions of low Doppler spread and low delay spread. Their performances degrade significantly when spreads exceed certain modem dependent values.

For the experiments undertaken by the University of Leicester, the DAMSON signals were received with large sampled aperture antenna arrays, each element of which was connected to a separate receiver. The complex amplitudes of the signals received on each antenna within the array were sampled simultaneously many times per second and the data processed to provide a measure of the relative times of flight of the propagating modes and their associated Doppler spectra. In this way, the signal was split into components distinguished by time of flight, Doppler frequency and by antenna position in the receiving array. A direction finding algorithm (a modified version of an iterative null steering super-resolution direction finding algorithm [25] based upon the IMP [26] and DOSE [27] algorithms) was then applied to each signal component in turn in order to estimate the directional characteristics of the received signal.

In addition to determining the channel scattering characteristics (the normal output of the DAMSON system), the use of the multi-channel receiving system enabled estimates to be made of the direction of arrival of each of the components of the received signal, separated by time of flight and Doppler frequency shift. It is evident from the DF measurements (see [22]) that there is a significant level of directional variation (see, for example, Figure 7) in the received signals over and above that which would normally be encountered due to multi-mode propagation effects at mid-latitudes (mainly elevational differences). In particular, these variations include deviations from the great circle path which may differ significantly between the various propagation modes and also directional spreading which is often related to the precise Doppler frequency within individual propagation modes. It is suggested in [23] that signals may be combined from two or more antennas in such a way as to, in effect, steer beam(s) or null(s) in the directional sensitivity pattern of the receiver antenna so as to exploit the directional diversity of the incident signals in order to reduce the delay and Doppler spreads at the modem input. Such a technique is potentially important for increasing the data throughput of communications systems employing modern, high speed modems operating within the difficult, high latitude environment.

\section{CONCLUDING REMARKS}

A wealth of knowledge resulted from the investigations into gross deviations from the great circle path (GCP) outlined above. The magnitude and variances of the deviations from the GCP have been quantified and the associated geophysical conditions and signal characteristics identified. Research is currently being undertaken aimed at incorporating the knowledge gained from the experiments into techniques that will be of direct operational application. The main activities in this area include the development of ionospheric models coupled with ray tracing studies (some preliminary results of which were presented here) both to confirm the cause of the large bearing deviations and to assess the impact of the presence of the high latitude ionospheric features causing the deviations on paths other than those subjected to experimental investigation. A set of rules (a 'rule-base') will be developed through which the influence of the off-great circle propagation mechanisms on specified communications links, radiolocation systems and OTH radars may be estimated. A further important activity will be the incorporation of the rule base into computer code and its integration with various prediction codes currently in use to allow proper consideration to be given to the off-great circle propagation mechanisms. 

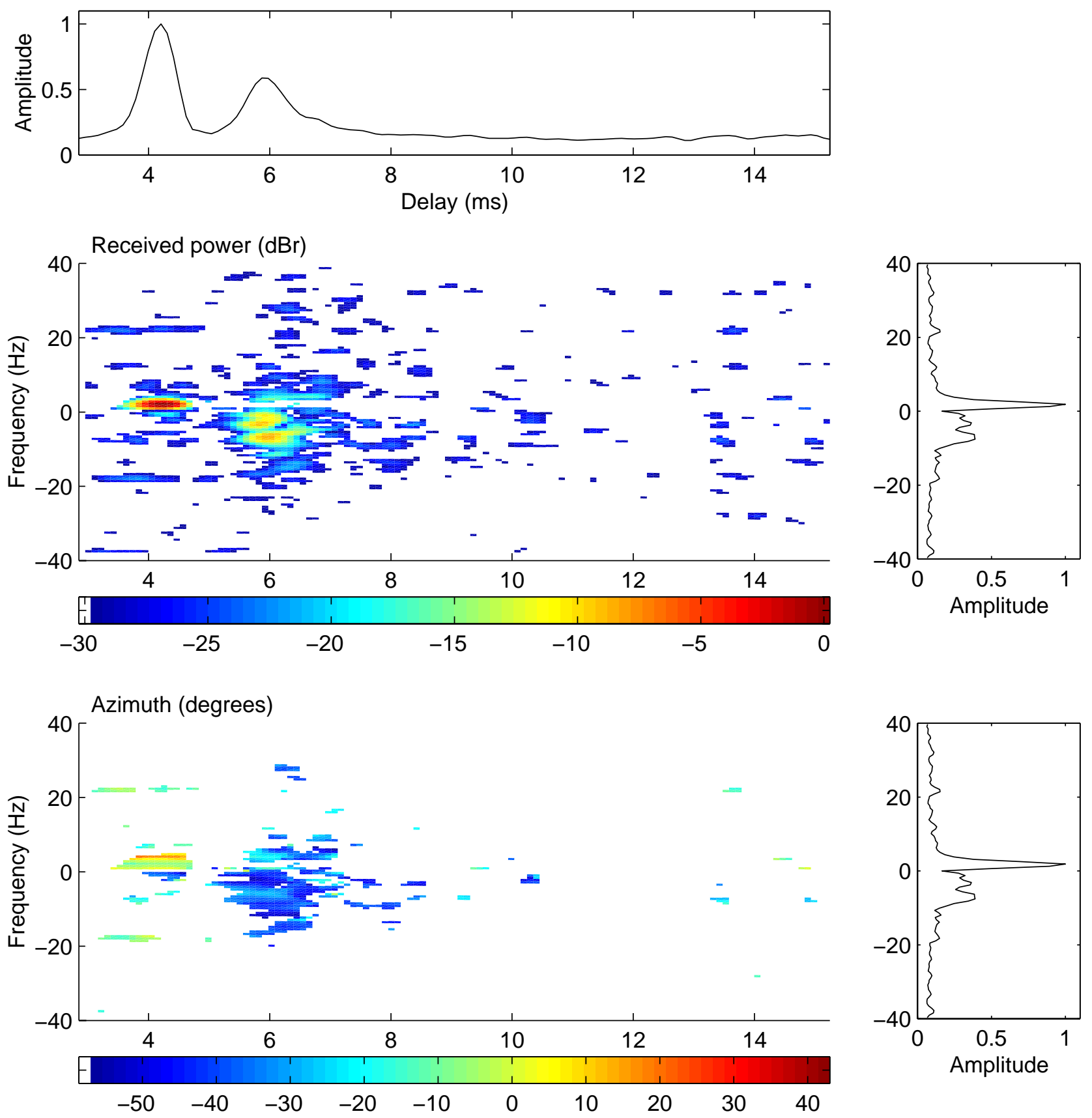

Figure 7. Delay-Doppler plot (upper colour frame) and bearings (lower colour frame) for the $4.7 \mathrm{MHz}$ signal received over the Isfjord to Kiruna path. 16 March 1998, 19:47 UT.

Further experimental studies are to be undertaken with a multi-channel receiver system installed at Kiruna, northern Sweden, and transmitters installed at Longyearbyen, Svalbard and at Kirkenes in northern Norway. The analysis of the data from the measurements will be directed towards (a) developing our knowledge and understanding of the complex propagation mechanisms prevalent in the high latitude regions, and (b) investigating the applicability of intelligent, adaptive beam / null steering (spatial filtering) to reduce the level of apparent Doppler and delay spreading and hence to increase the available data throughput. The increased understanding of the propagation mechanisms and the signal characteristics will be employed in developing physically realistic models incorporating directional information of signal behaviour as a function of frequency, time of day, time of year and geophysical conditions 
A further important aspect of this investigation will be to investigate the relationships between the oblique propagation characteristics and the CUTLASS [28] ionospheric radar observations over an extended period. The identification of suitable well-defined relationships will enable the estimation of oblique signal characteristics for prolonged periods (i.e. those periods not covered by these experiments but covered by the other instrumentation) and over other paths by examination of the data produced by the continuously operating geophysical instrumentation. It may therefore be possible to extend the results of this work to encompass the extensive geographical area covered by the SuperDARN radar network, of which the CUTLASS radars form an element.

\section{ACKNOWLEDGEMENTS}

The work undertaken at the University of Leicester outlined above was undertaken by a number of researchers, both past and present. The authors would like to acknowledge their contributions to this work their names can be determined from the citations given in the text. Mention was also made of measurements made of the signals radiated from the DAMSON system, The authors would like to thank the UK Defence Evaluation Research Agency, the Canadian Communications Research Centre, the Norwegian Defence Research Establishment, the Swedish Institute of Space Physics and the Swedish National Defence Research Establishment for their help in using these transmissions.

\section{REFERENCES}

1 Jones, T.B. and Reynolds, J.S.B. Ionospheric perturbations and their effect on the accuracy of HF direction finders. Radio and Electronic Engineer, 45, 1975, pp 63-73.

2 Rogers, N.C., Warrington, E.M. and Jones, T.B. Large HF bearing errors for propagation paths tangential to the auroral oval. IEE Proceedings on Microwaves, Antennas and Propagation, 144, (2), 1997, pp 91-96.

3 Stocker, A.J., Warrington, E.M. and Jones, T.B. Directions of arrival at three frequencies on a propagation path along the mid-latitude trough: A comparison of observations with ray tracing simulations. In: Proceedings of the IEE Eighth International Conference on HF Systems and Techniques, 10-13 July 2000, pp 193-197.

4 Stocker, A.J., Warrington, E.M. and Zaalov, N.Y. The effect of the mid-latitude trough on the direction of arrival and time-of-flight of HF radio signals. Ibid.

5 Warrington, E.M. Rogers, N.C. and Jones, T.B. Large HF bearing errors for propagation paths contained within the polar cap. IEE Proceedings on Microwaves, Antennas and Propagation, 144, (4), 1997, pp 241-249.

6 Warrington, E.M. Observations of the directional characteristics of ionospherically propagated HF radio channel sounding signals over two high latitude paths. IEE Proceedings on Microwaves, Antennas and Propagation, 145, (5), 1998, pp 379-385.

7 Warrington, E.M., Jackson, C.A., Stocker, A.J., Jones, T.B. and Lundborg, B. Observations of the directional structure of obliquely propagating HF radio signals and simultaneous HF radar measurements - a case study. In: Proceedings of the IEE Eighth International Conference on HF Systems and Techniques, 10-13 July 2000, pp 243-247.

8 Warrington, E.M., Jackson, C.A. and Lundborg, B. Directional diversity of HF signals received over high latitude paths and the possibility of improved data throughput by means of spatial filtering. IEE Proceedings on Microwaves, Antennas and Propagation, 147, (6), 2000.

9 Weber, E.J., Buchau, J., Moore, J.G., Sharber, J.R., Livingston, J.D., Winningham, J.D., and Reinische, B.W. F layer ionization patches in the polar cap. Journal of Geophysical Research, 89, 1984, pp 1683-1694.

10 Buchau, J., Reinisch, B.W., Weber, E.J. and Moore, J.G. Structure and dynamics of the winter polar cap F region. Radio Science, 18, (6), 1983, pp 995-1010.

11 Carlson, H.C. Jr., Wickwar, V.B., Weber, E.J., Buchau, J., Moore, J.G. and Whiting, W., Plasma characteristics of polar cap F layer arcs. Geophysical Research Letters, 11, 1984, pp 895-898.

12 Gussenhoven, M.S. Extremely high latitude auroras. Journal of Geophysical Research, 87, 1982, pp 2401-2412.

13 Holzworth, R.H. and Meng C.-I. Mathematical representation of the auroral oval. Geophysical Research Letters, 2, (9), 1975, pp 377-380. 
14 Lockwood, M. Modelling the high latitude ionosphere for time varying plasma convection. Proceedings of the IEE, part H, 140, (2), 1993, pp 91-100.

15 Jones R.M. and Stephenson J.J. A Versatile Three-Dimensional Ray Tracing Computer Program for Radio Waves in the Ionosphere. Office of Telecommunications, OT 75-76, U.S Department of Commerce, Washington, USA, 1975.

16 Bilitza, D. (ed.). International Reference Ionosphere 1990. NSSDC 90-22, Greenbelt, Maryland, USA, 1990.

17 N.C. Rogers, Warrington, E.M. and Jones, T.B.. Oblique ionogram features associated with off-greatcircle HF propagation at high latitudes. In: The Proceedings of the IEE Eleventh International Conference on Antennas and Propagation, Manchester, April 2001, pp 398-402.

18 Warrington, E.M., Stocker, A.J. and Rizzo, C. Behaviour of superresolution direction finding algorithms for $\mathrm{HF}$ signals propagating through the high latitude ionosphere. IEE Proceedings on Microwaves, Antennas and Propagation, 147, (6), 2000, pp 469-478.

19 Dumas, D.J. High-latitude HF direction finding: a case study and modelling results. In: The Proceedings of the Second Symposium on Radiolocation and Direction Finding, Southwest Research Institute, San Antonio, 4-6 November 1997, (29 pages).

20 Featherstone, W., Strangeways, H.J., Zatman, M.A. and Mewes, H. A novel method to improve the performance of Capon's minimum variance estimator. In: The Proceedings of the IEE Tenth International Conference on Antennas and Propagation, IEE Conference Publication 436, 1997, pp 1.322-1.325.

21 Read, W.J.L. Advanced DF algorithms for high latitude operation. In Proceedings of the Third Symposium on Radiolocation and Direction Finding, Southwest Research Institute, San Antonio, 24 November 1999, (33 pages).

22 Warrington, E.M. Observations of the directional characteristics of ionospherically propagated HF radio channel sounding signals over two high latitude paths. IEE Proceedings on Microwaves, Antennas and Propagation, 145, (5), 1998, pp. 379-385.

23 Warrington, E.M., Jackson, C.A. and Lundborg, B. Directional diversity of HF signals received over high latitude paths and the possibility of improved data throughput by means of spatial filtering. IEE Proceedings on Microwaves, Antennas and Propagation, 147, (6), 2000, pp 487-494.

24 Davies, N.C. and Cannon, P.S. DAMSON - A system to measure multipath dispersion, Doppler spread and Doppler shift on a multi-mechanism communications channels. In: Proceedings of AGARD EPP Symposium on Multiple Mechanism Propagation Paths: Their Characterisation and Influence on System Design, Rotterdam, October 1993, pp 36/1-6.

25 Warrington, E.M. Measurements of the direction of arrival of HF sky wave signals by means of a wide aperture antenna array and two super-resolution direction finding algorithms. IEE Proceedings on Microwaves, Antennas and Propagation, 142, (2), 1995, pp 136-144.

26 Mather, J. The incremental multi-parameter algorithm. Asilomar conference on circuits, systems and computers, Monterey, 1990. Published by Maple Press, San Jose, California, USA, 1, 1991, pp 368-372.

27 Zatman, M., Strangeways, H.J. and Warrington, E.M. Resolution of multimoded HF transmissions using the DOSE superresolution direction finding algorithm. In: Proceedings of the IEE Eighth International Conference on Antennas and Propagation, IEE Conference Publication 370, 1993, pp 415-418.

28 Jones, T.B. and Thomas, E.C. CUTLASS: A new HF auroral radar. In: Proceedings of the IEE Seventh International Conference on HF Radio Systems and Techniques, IEE conference publication number 441, July 1997, pp 325-329. 